\title{
Theory of Nickel and and Nickel-Hydrogen Complexes in Silicon
}

\author{
R. Jones \\ Department of Physics, University of Exeter, Exeter, EX\&4QL, United Kingdom. \\ S. Öberg \\ Department of Mathematics, University of Luleà, Luleà, S95 187, Sweden \\ J. Goss \\ Department of Physics, University of Exeter, Exeter, EX4 4QL, United Kingdom. \\ P. R. Briddon \\ Department of Physics, University of Newcastle-upon-Tyne, Newcastle-upon-Tyne, NE1 7RU, \\ United Kingdom.
}

A. Resende

Departamento de Física, Universidade de Aveiro, 3800 Aveiro, Portugal

\begin{abstract}
Spin-polarized local density functional cluster calculations are carried out on substitutional $\mathrm{Ni}$ and $\mathrm{Ni}-\mathrm{H}_{2}$ complexes. We find that $\mathrm{Ni}^{-}$undergoes a JahnTeller distortion along $\langle 100\rangle$ with Ni moving slightly along the cube axis. The distorted state gives $b_{1}, b_{2}$ and $a_{1}$ levels in ascending order of energies within the gap in agreement with experiment. Several candidate $\mathrm{NiH}_{2}$ defects are investigated: the lowest energy structure consists of a substitutional Ni atom together with two $\mathrm{H}$ atoms at anti-bonding sites to two Si neighbors of Ni. This gives $H$ related vibrational modes and a spin-polarized charge density close to those reported for $\mathrm{Pt}-\mathrm{H}_{2}$.
\end{abstract}


The vacancy model of substitutional transition metal (TM) impurities on the far-right of the periodic table $[1,2]$, considers that the $d$ levels of the TM impurity lie deep in the valence band of Si and couple weakly with the $t_{2}$ levels arising from the $T_{d}$ symmetry of the ideal vacancy. The important implication is then that the gap-levels and structure of the defect are largely determined by those of a perturbed vacancy [3-6]. For $\mathrm{Pt}^{-}, \mathrm{Pd}^{-}$and $\mathrm{Ni}^{-}$, the gap $t_{2}$ levels contain three electrons and according to the Jahn-Teller theorem, a distortion will occur for an $S=1 / 2$ state, leading to a splitting of these levels and a lowering of energy. Deep level transient spectroscopy shows that $\mathrm{Ni}_{s}$ possesses acceptor and donor activation energies at $E_{c}-0.45$ and $E_{\imath}+0.16 \mathrm{eV}$ respectively [7]. Electron paramagnetic resonance (EPR) measurements on $\mathrm{Ni}^{-}[8]$ and $\mathrm{Pt}^{-}[2,9]$ reveal that the defect has $C_{2 v}$ symmetry and furthermore, uniaxial stress measurements on the EPR lines have shown $[9,10]$ that the gap $t_{2}$ levels split into a configuration $b_{1}^{\uparrow \downarrow} b_{2}^{\uparrow} a_{1}^{0}$. This ordering of levels is the reverse to that found for the negatively charged vacancy. Further evidence for the vacancy model has been suggested recently when it was found that Pt-H complexes can be created by high temperature in-diffusion of the impurities [11-14]. Two localized vibrational modes (LVMs) attributed to $\mathrm{Si}-\mathrm{H}$ have been observed which undergo small shifts in mixed H-D implantation or when the charge state of the defect is changed. These results establish the presence of more than one $\mathrm{H}$ in the defect. The first suggestion was that the $\mathrm{H}$ atoms bond to two of the four Si atoms surrounding the vacancy. It is the purpose of this work to investigate this model.

As far as we have been aware, there have been no $a b$ initio calculations of the JahnTeller distortion or the splitting of the $t_{2}$ levels, nor of the complexes with $H$. We report here local density functional spin-polarized calculations on substitutional nickel, $\mathrm{Ni}_{s}$, and $\mathrm{Ni}-\mathrm{H}_{2}$ complexes using large (up to 133 atoms) H-terminated clusters. A key ingredient is that we allow the atoms surrounding the defect to move and this enables us to investigate in detail the Jahn-Teller distortion due to $\mathrm{Ni}_{s}^{-}$and to obtain the structure and the LVMs of the $\mathrm{Ni}-\mathrm{H}_{2}$ defect. The LVMs are found by two methods. In the first we numerically differentiated the forces on $\mathrm{H}$ and the surrounding atoms and these, together with a pre- 
viously determined potential, were used to construct the dynamical matrix of the cluster. This gives quasi-harmonic LVMs. In the second method [16], the energies corresponding to displacing the H atoms from their equilibrium sites were found and the Schrödinger equation for the oscillator solved numerically using these energies as a potential in accordance with the Born-Oppenheimer approximation. The two methods give rather similar results.

The $a b$ initio method has been described previously [17] and it has been adapted for the present study to deal with $d$ orbitals and spin-polarization effects $[18,19]$. We investigate the case of $\mathrm{Ni}$ since spin-orbit coupling is less important in this case than for Pd and Pt. The electronic wavefunctions of the cluster were expanded in atom-sited Gaussian orbitals. Seven independent Gaussian s,p,d orbitals with different exponents were placed on the Ni atom, two on each $\mathrm{H}$ atom and four on the central four Si atoms. The remaining basis consisted of fixed linear combinations of the $s$ and $p$ orbitals with different exponents and these are centered on the other atoms. The charge density was fitted to a linear combination of $s^{-}$ Gaussian functions: 14, 5 and 3 sited on Ni, Si and H respectively. Ni and Si pseudopotentials were taken from Ref. [20]. We have shown that the method describes small molecules containing $\mathrm{Ni}$ very accurately: the bond lengths of $\mathrm{Ni}(\mathrm{CO})_{4}$ for example are found to be within $1 \%$ of their experimental values. The cluster approach for H defects in bulk Si locates LVMs to within about $100 \mathrm{~cm}^{-1}[21]$.

We investigated $\mathrm{Ni}_{s}^{-}$embedded in a 71 atom cluster, $\mathrm{NiSi}_{34} \mathrm{H}_{36}$. The $\mathrm{Ni}$ atom was placed along [100] so that the symmetry of the cluster is $C_{2 v}$. Three close-by levels for each spin lay in the gap region and these are occupied with three electrons according to fermi-statistics corresponding to $0 \mathrm{~K}$, i. e. the lowest was filled, the next filled in the up-spin case only and the others were empty. The self-consistent energy and forces acting on the central 17 atoms were then found for this $S=1 / 2$ configuration, and these atoms were allowed to move to minimize the energy of the cluster using a conjugate gradient algorithm. The Ni atom moved closer to the substitutional site while the surrounding Si atoms moved outwards from their lattice positions. The splitting between the $t_{2}$-derived levels gradually decreased and eventually we were unable to obtain a self-consistent solution. This arises because during 
the course to self-consistency, the three close-by gap levels, the uppermost of which is empty, cross over and if, for example, the $a_{1}$ state falls below the $b_{1}$ state, the charge density and hence potential changes discontinuously. On the next iteration, the level ordering often reverses and the process never converges. This problem of 'charge sloshing' is well-known and often occurs for close-by levels. It can be overcome by occupying the gap-levels with fermi-statistics corresponding to a finite temperature larger than the splitting of the $t_{2}$ levels. This spreads out the electrons among the three gap levels and the discontinuity in the charge density arising from cross-over is reduced. However, this almost eliminates the Jahn-Teller driving force for the $\mathrm{Ni}$ atom to lie off-site. For the finite-temperature calculation the lowest energy configuration corresponded to a structure where the $\mathrm{Ni}$ atom is essentially on site but the four surrounding Si atoms were displaced outwards from their lattice sites by 0.270 $\AA$ and two of them were further displaced along $[100]$ by $0.006 \AA$. Second shell Si atoms were displaced by $\approx 0.07 \AA$. The three gap levels were split by only $0.005 \mathrm{eV}$ and the highest occupied spin-up level was $0.6 \mathrm{eV}$ below the corresponding (but empty) spin-down level. Thus the departure from $T_{d}$ symmetry is extremely small. The ordering of the $t_{2}$-derived gap levels is $b_{1}^{\uparrow \downarrow} b_{2}^{\uparrow} a_{1}^{0}$ is the same as that found experimentally.

The problem of obtaining self-consistent solutions can now be overcome by restricting the electronic configuration to be $b_{1}^{\uparrow \downarrow} b_{2}^{\uparrow} a_{1}^{0}$. Then during the passage to self-consistency, there is no discontinuity in the charge density but the energy levels that are occupied at each stage are not necessarily in ascending order. However, towards the end of the self-consistency cycle the energy levels ordered correctly and the final energy obtained was lower than that obtained by the imposition of a temperature. The Ni atom was then moved along [100] and the equilibrium structure was found to correspond to a displacement of $0.13 \AA$ from the lattice site. The two Si atoms with cartesian components along [100] were displaced almost radially by $(0.15, \pm 0.25, \pm 0.22) \AA$ and moved away from each other, whereas the other two Si atoms with components along [100] were displaced $(-0.07, \pm 0.16, \mp 0.13) \AA$ so that they approached each other. Curiously, the four Ni-Si bonds are almost identical in length at $2.645 \AA$. This distortion is similar to that found experimentally for the negatively charged 
vacancy [22].

Suppose now, we occupy the $t_{2}$ derived states according to $a_{1}^{\uparrow \downarrow} b_{1}^{\uparrow} b_{2}^{0}$. This is the configuration found experimentally for the negatively charged vacancy [22] but not for $\mathrm{Ni}_{s}^{-}$. Then the self-consistent cluster energy is higher than the previous configuration by $0.017 \mathrm{eV}$. We note that although we have shown that the $C_{2 v}$ distortion has a lower energy than $T_{d}$ it leaves open the possibility that other symmetries, e. g. $D_{2}$ have even lower energies as suggested in Ref. [15]. The slight distortion from $T_{d}$ to $C_{2 v}$ symmetry is consistent with the ease of reorientation of $\mathrm{Pt}$ around the central lattice site. This motion occurs for temperatures above $12 \mathrm{~K}$ or even when the slightest stress is imposed on the crystal at $2 \mathrm{~K}[9]$.

The pseudo-wave function of the $t_{2}$-derived levels all possess a nodal surface lying between the TM impurity and the surrounding Si atoms and peaks on either side of the Si atoms along $\langle 111\rangle$. This suggests that $\mathrm{H}$ atoms will lie either between $\mathrm{Si}$ and $\mathrm{Ni}$ in the configuration suggested previously [10,11,13,14], or outside at anti-bonding (AB) sites as shown in Fig. 1. The effect of $\mathrm{H}$ was investigated in a 133 atom negatively charged cluster $\mathrm{NiSi}_{70} \mathrm{H}_{62}$ where 19 central atoms were relaxed. There were no problems with self-consistency in the present case. For the first defect, the $\mathrm{H}$ atoms repelled $\mathrm{Ni}$ along [100] from its lattice site by $0.20 \AA$ and formed $\mathrm{Si}-\mathrm{H}$ bonds of length $1.508 \AA$ with a H-H separation of $1.517 \AA$. The LVMs due to $\mathrm{H}$ were calculated to lie at 2477 and $2511 \mathrm{~cm}^{-1}$. These values are far from the experimental ones for $\mathrm{PtH}_{2}[10-12]$ and strongly suggest that this model is incorrect. The strong coupling between the $\mathrm{H}$ atoms occurs because there is so little room for both $\mathrm{Ni}$ and $H$ within the vacancy. The second defect (Fig. 1) when relaxed yielded an energy 0.08 $\mathrm{eV}$ below the first structure and the Ni atom moved $0.3 \AA$ along [100] towards the H atoms and the $\mathrm{Si}-\mathrm{H}$ lengths became $1.557 \AA$. The separation between the $\mathrm{H}$ atoms was $7.3 \AA$. The vibrational modes are given in the Table. The two $\mathrm{H}$ related modes are slightly split, by 7 $\mathrm{cm}^{-1}$, with the higher mode being $A_{1}$. Now, uniaxial stress splitting experiments on $\mathrm{PtH}_{2}$ [12] show that the upper and lower modes also have $A_{1}$ and $B_{1}$ symmetries respectively. The stretch modes are close to those reported for $\mathrm{PtH}_{2}$ but the bend modes have not yet been detected. 
Anharmonic effects are known to be important for $\mathrm{H}$ modes [16,23,24] and we investigated their influence in the following way: the two modes at 2009.9 and $2003.2 \mathrm{~cm}^{-1}$ represent $A_{1}$ and $B_{1}$ vibrational modes in which the $\mathrm{H}$ atoms move either in phase or out of phase with each other. The energies necessary to displace the H atoms by an amount $r$, parallel to the Si-H bonds, were evaluated for each of these modes. This energy contains only even powers of $r$ in the $B_{1}$ mode whereas it contains both even and odd powers in the $A_{1}$ mode. The Schrödinger equation for the oscillator was solved numerically [16] and the $A_{1}$ and $B_{1}$ frequencies were then found to be 2037 and $2060 \mathrm{~cm}^{-1}$ and are separated by 23 $\mathrm{cm}^{-1}$. However, now the $B_{1}$ mode lies above the $A_{1}$ mode contrary to experiment. Thus even though the separation of the $\mathrm{H}$ atoms is very large, $7.3 \AA$, the two modes are split and their ordering reversed by these anharmonic effects by an amount twice as large as that observed for $\mathrm{Pt}-\mathrm{H}_{2}$. However, there are other anharmonic terms which should be considered. For example, $r_{1} \theta_{1}^{2}+r_{2} \theta_{2}^{2}$ terms where $r_{k}$ and $\theta_{k}$ are the changes in the equilibrium $\mathrm{Si}_{k}-\mathrm{H}_{k}$ lengths and angles respectively. These mix the stretch and bend modes and are only present in the $A_{1}$ mode where $r_{1}=r_{2}$. These have the effect of decreasing the mean Si-H length hence pushing up the $A_{1}$ frequency. Such a term then might well displace the $A_{1}$ mode above that of $B_{1}$.

We investigated several other models of the defect but all gave energies above that of the AB structure. In addition, their vibrational modes were far from the observed values and often, the $B_{1}$ mode lay higher than that of the $A_{1}$. We suggest then that the $H$ atoms lie at $\mathrm{AB}$ sites in the $\mathrm{Pt}$ case especially as its larger size makes it even less likely that $\mathrm{H}$ would lie inside.

Further arguments against the configuration in which $\mathrm{H}$ atoms are inside the vacancy come from the comparison with LVMs assigned to the vacancy-hydrogen, $\mathrm{VH}_{2}$, and $\mathrm{H}_{2}^{*}$ complexes. The former are observed at 2122 and $2144 \mathrm{~cm}^{-1}$ [25]. The separation between the $\mathrm{H}$ modes here, $\approx 23 \mathrm{~cm}^{-1}$, is larger than that observed in $\mathrm{PtH}_{2}$ where it might be expected that the TM squeezes the H atoms together, increasing their interaction and the mode splitting. In the case of $\mathrm{H}_{2}^{*}$, the $\mathrm{AB}$ sited $\mathrm{H}$ atom gave an LVM at $1838 \mathrm{~cm}^{-1}$ [21] 
rather close to the stretch mode of the $\mathrm{Pt} \mathrm{H}_{2}$ complex, whereas the other H-LVM, attributed to H-Si stretch where Si has a tetrahedral environment, has a frequency at $2061.5 \mathrm{~cm}^{-1}$ - somewhat higher than that found for $\mathrm{Pt}-\mathrm{H}_{2}$. Recently, Uftring et al [26] analyzed the anisotropic hyperfine parameter in $\mathrm{PtH}_{2}$ and concluded that the $\mathrm{Pt}-\mathrm{H}$ distance is about $4.5 \AA$ which is close to the calculated Ni-H length of $4.28 \AA$ in the $\mathrm{AB}$ sited model.

The wave-function for the highest occupied level in $\mathrm{NiH}_{2}^{-}$(AB) has $b_{2}$ symmetry and vanishes in the (011) plane containing the two H atoms. This level occurs around mid-gap in our calculations and the next lower level is very close to the valence band. The positions of these levels is only approximate but they suggest that the observed EPR signals are due to $\mathrm{PtH}_{2}^{-}$complexes, for it is known that when $E_{f}$ lies above $E_{c}-0.1 \mathrm{eV}$, then the defect is not paramagnetic. There must be a second acceptor level corresponding to the filling of $b_{2}^{\downarrow}$ around $E_{c}-0.1 \mathrm{eV}$ and leading to diamagnetic $\mathrm{NiH}_{2}^{--}$. Uftring et al [26] also concluded that the defect has two acceptor levels as the effect of illumination on the paramagnetic complex is easily understood to arise from the capture of photo-generated holes by $\mathrm{PtH}_{2}^{-}$. In addition, they suggested that the $\mathrm{Pt}$ hyperfine data is consistent with the $\mathrm{H}$ atoms lying in the nodal plane of the highest occupied level. For $\mathrm{NiH}_{2}^{-}$, the contour plots of the pseudowavefunctions suggest that the isotropic hyperfine interaction with $H$ would vanish leaving only an anisotropic one. However, spin-polarization causes a difference in all the spin-up and spin-down valence wave-functions and results in a small polarized charge density of -0.007 a. u. at each proton. This is only $-2 \%$ of the charge density of a $H$ atom in vacuo. Its magnitude is within a factor of three found experimentally for $\mathrm{PtH}_{2}[10-12,14]$. It is unclear whether this discrepancy is due to calculational errors or differences between $\mathrm{Ni}$ and $\mathrm{Pt}$.

In conclusion, the calculations show that the substitutional $\mathrm{Ni}^{-}$defect with $T_{d}$ symmetry is unstable against a displacement along the $\langle 100\rangle$ axis. The $t_{2}$ gap level is split into $b_{1}, b_{2}$ and $a_{1}$ levels in ascending energy. $\mathrm{Ni}_{s}$ can complex with two $\mathrm{H}$ atoms at $\mathrm{AB}$ sites and act as a double acceptor. It gives two H-LVMs around $2000 \mathrm{~cm}^{-1}$. The polarization charge density is very small and negative at the $\mathrm{H}$ nuclei and we suggest that $\mathrm{PtH}_{2}$ complexes assume the same structure. 
S. Ö. thanks the Swedish National Scientific Research Council, for financial support. R. J. thanks G. D. Watkins, M. J. Stavola and S. Uftring for useful discussions. The authors also thank the HPCI for computer time on the T3D where some of the calculations were performed.

\section{REFERENCES}




\section{REFERENCES}

[1] G. D. Watkins, Physica, 117-118B, 9 (1983).

[2] F. G. Anderson, R. F. Milligan, and G. D. Watkins, Phys. Rev. B 45, 3279 (1992).

[3] L. A. Hemstreet, Phys. Rev. B 15, 834 (1977).

[4] A. Zunger, and V. Lindefelt, Phys. Rev. B 27, 1191 (1983).

[5] J. L. A. Alves, and J. R. Leite, Phys. Rev. B 30, 7284 (1984).

[6] M. Lannoo, Phys. Rev. B 36, 9355 (1987).

[7] H. Nakashima, T. Sadoh, H. Kitagwa, and K. Hashimoto, Mater. Sci. Forum, 143-7, 761 (1994).

[8] L. S. Vlasenko, N. T. Son, A. B. van Oosten, C. A. J. Ammerlaan, A. A. Lebedev, E. S. Taptygov, and V. A. Khramtov, Solid State Comm. 73, 393 (1990).

[9] F. G. Anderson, F. S. Ham, and G. D. Watkins, Phys. Rev. B 45, 3287 (1992).

[10] P. M. Williams, F. S. Ham, F. G. Anderson, and G. D. Watkins, Mater. Sci. Forum, 83-7, 233 (1992).

[11] P. M. Williams, G. D. Watkins, S. Uftring, and M. Stavola, Phys. Rev. Lett. 70, 3816 $(1993)$.

[12] P. M. Williams, G. D. Watkins, S. Uftring, and M. Stavola, Mater. Sci. Forum, 143-7, 891 (1994).

[13] M. Höhne, U. Juda, Yu. V. Martynov, T. Gregorkiewicz, C. A. J. Ammerlaan, and L. S. Vlasenko, Mater. Sci. Forum, 143-7, 1659 (1994).

[14] M. Höhne, U. Juda, Yu. V. Martynov, T. Gregorkiewicz, C. A. J. Ammerlaan, and L. S. Vlasenko, Phys. Rev. B 49, 13423 (1994).

[15] F. G. Anderson, F. S. Ham, and G. Grossman, Mater. Sci. Forum 83-7, 475 (1992). 
[16] R. Jones, J. Goss, C. Ewels, and S. Öberg, Phys. Rev. B 50, 8378-88, (1994).

[17] R. Jones, Phil. Trans. Roy. Soc. London Ser. A 341, 351 (1992).

[18] G. M. S. Lister, and R. Jones, unpublished report, 1988.

[19] P. R. Briddon, and R. Jones, unpublished.

[20] G. B. Bachelet, D. R. Hamann and M. Schlüter, Phys. Rev. B 26, 4199 (1982).

[21] J. D. Holbech, B. Bech Nielsen, R. Jones, P. Sitch, and S. Öberg, Phys. Rev. Lett. 71, $875(1993)$.

[22] G. D. Watkins, in Deep Centers in Semiconductors, edited by S. T. Pantelides, (Gordon and Breach, New York, 1986), p 147.

[23] R. C. Newman, Physica B 170, 409 (1991).

[24] R. Darwich, B. Pajot, B. Rose, D. Robein, B. Theys, R. Rahbi, C. Porte, and F. Gendron, Phys. Rev. B 48, 17776 (1993).

[25] B. Bech Nielsen, L. Hoffmann, M. Budde, R. Jones, J. Goss, and S. Öberg, unpublished.

[26] S. J. Uftring, M. Stavola, P. M. Williams, and G. D. Watkins, Phys. Rev. B 51, 9612 $(1995)$.

[27] S. Uftring, private communication. 


\section{TABLES}

TABLE I. Vibrational Modes, $\mathrm{cm}^{-1}$, for the TM- $\mathrm{H}_{2}^{-}$AB defect

\begin{tabular}{|c|c|c|c|c|c|c|}
\hline Calc. & $A_{1}$ & $B_{1}$ & $A_{2}$ & $B_{2}$ & $A_{1}$ & $B_{1}$ \\
\hline $\mathrm{Ni} \mathrm{H}_{2}$ & 2009.9 & 2003.2 & 903.0 & 897.2 & 890.4 & 889.4 \\
\hline $\mathrm{NiH} \mathrm{D}$ & 2006.6 & 1442.5 & 900.1 & 889.8 & 636.6 & 630.6 \\
\hline $\mathrm{Ni} \mathrm{D}_{2}$ & 1445.1 & 1439.9 & 638.7 & 634.6 & 631.6 & 629.5 \\
\hline \multicolumn{7}{|l|}{$\operatorname{Expt}^{a, b}$} \\
\hline $\mathrm{Pt} \mathrm{H}_{2}$ & 1901.6 & 1888.2 & & & & \\
\hline Pt HD & 1894.6 & 1366.9 & & & & \\
\hline $\mathrm{Pt} \mathrm{D}_{2}$ & 1370.7 & 1362.5 & & & & \\
\hline
\end{tabular}

${ }^{a}$ Ref. $[11,12]{ }^{b}$ Ref. [27] 


\section{FIGURES}

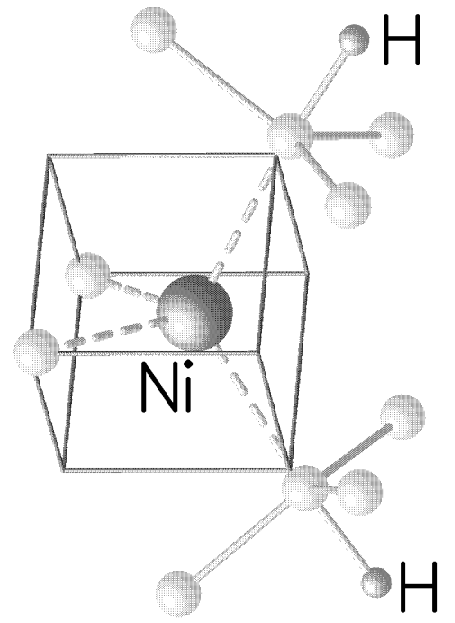

FIG. 1. Ni- $\mathrm{H}_{2}$ complex with $\mathrm{H}$ at the anti-bonding (AB) sites. 